\title{
The benefits of psychosocial interventions for cancer patients undergoing radiotherapy
}

\author{
Zhen Guo ${ }^{1 \dagger}$, Hua-ying Tang ${ }^{1,2 \dagger}$, Hao Li ${ }^{3}$, Sheng-kui Tan ${ }^{4}$, Kai-hua Feng ${ }^{1}$, Yin-chun Huang ${ }^{1}$, Qing Bu ${ }^{5}$ \\ and Wei Jiang ${ }^{1 *}$
}

\begin{abstract}
Background: Many patients with cancer experience depression and anxiety, and an associated decrease in quality of life (QOL) during radiation therapy (RT). The main objective of the study was to determine the benefits of psychosocial interventions for cancer patients who received RT.

Methods: Patients with cancer $(n=178)$ who agreed to participate in the study were randomized to the intervention arm $(n=89)$ or the control arm $(n=89)$. Patients in the intervention group received psychosocial care during RT, whereas the control group received RT only. The benefits of the intervention were evaluated using the Zung Self-rating Depression Scale (SDS) to measure depression, the Self-rating Anxiety Scale (SAS) to assess anxiety, and the European Organization for Research and Treatment of Cancer Quality of Life Questionnaire-Core 30 (EORTC QLQ-C30) to survey health-related QOL. The association between intervention and survival was also assessed.
\end{abstract}

Results: Patients randomly assigned to the intervention arm showed significant improvements on symptoms of depression $(p<0.05)$ and anxiety $(p<0.05)$, health-related QOL $(p<0.05)$ (i.e. better global health status, and physical and emotional functioning, and less insomnia) when compared with controls. In the subset analysis, female patients, those that received high dose irradiation, and those that underwent adjuvant chemotherapy could benefit more from psychosocial intervention. There was no difference between the two groups in disease-free survival (DFS) (2-year DFS 79.8\% in the intervention arm and $76.4 \%$ in the control arm; $p=0.527$ ) and overall survival (OS) (2-year OS $83.1 \%$ in the intervention arm and $84.3 \%$ in the control arm; $p=0.925$ )

Conclusions: Psychosocial intervention is a cost-effective approach that can improve a patient's mood and QOL both during and after RT. However, the intervention was not found to reduce the risk of cancer recurrence and death.

Trial registration: ChiCTR-TRC-12002438

Keywords: Cancer, Radiation oncology, Psychosocial intervention, Anxiety, Depression, Quality of life

\section{Background}

During the course of cancer treatment, about two-thirds of patients will undergo radiation therapy (RT) as an essential component of a treatment program aimed at curing the disease, prolonging life or palliating symptoms [1] . However, RT often has a strong negative impact on cancer patients: it commonly leads to long-term physical effects(e.g. pain, and decreased physical functioning) and

\footnotetext{
* Correspondence: weijiang@glmc.edu.cn

${ }^{\dagger}$ Equal contributors

'Department of Radiation Oncology, Guilin Medical College Affiliated Hospital, 15 Lequn Road, Guilin, People's Republic of China

Full list of author information is available at the end of the article
}

emotional distress (e.g. anxiety and depression) [2]. Various studies have demonstrated that anxiety and depression are important and prevalent problems [3,4] that affect QOL in patients with cancer [5-8]; they also reduce compliance with treatment and prolong hospitalization $[9,10]$, and can compound the physical consequences of the disease [11,12]. Studies have shown that about $41 \%$ of tumor patients need professional psycho-oncological support [13], but less than $10 \%$ of patients are referred for psychosocial intervention in clinical practice daily [14].

Although a psychological burden associated with cancer is common, it is not inevitable. Psychosocial interventions have been shown to be effective in reducing

\section{Ciomed Central}


distress in cancer patients. For example, Osborn analyzed 15 randomized controlled trials that investigated the effects of psychosocial intervention on commonly reported problems such as depression, anxiety, pain, physical functioning, and QOL in adult cancer survivors, and reported that individual interventions were more effective than a control group. The interventions were found to reduce emotional distress and improving QOL in those surviving cancer [15]. Similarly, a recent metaanalysis by Sheinfeld and colleagues reviewed 37 papers (which included 4199 participants), with pain severity and interference as primary outcome measures. The analysis found that psychosocial interventions had mediumsize effects on both pain severity and interference, and suggested that such interventions should be a part of a multimodal approach to the management of pain in patients with cancer [16]. Further benefits of psychosocial interventions were found in extensive studies. Such psychological interventions were regarded as an inexpensive way to reduce psychological distress [17] and possibly to improve immune system functioning $[18,19]$ and prolong survival in patients with cancer [20].

However, few studies have compared emotional state and QOL in those receiving psychosocial intervention and a control group in cancer populations undergoing RT. The goal of this study was to adapt a randomized parallel control design to investigate whether a psychosocial intervention before and during RT could reduce emotional distress and improve treatment outcomes, including measures of QOL in patients newly diagnosed with cancer.

\section{Methods}

\section{Design}

The study will evaluate the benefits of psychosocial interventions for cancer patients within the period of RT through a two-armed randomized controlled trial (Clinical trial registration: ChiCTR-TRC-12002438; http:// apps.who.int/trialsearch/ Trial.aspx? TrialID = ChiCTRTRC-12002438.) Participants were randomized to one of two groups: RT alone (control group) or a psychosocial intervention plus RT. The status of patients was measured during the period between confirmation of the diagnosis and the start of RT, and again two weeks after the completion of RT. All patients were followed up over 2 years (Figure 1). The intervention was carried out by specially trained conductors. All data were collected through self-administered questionnaires. Ethics approval had been obtained from Guilin Medical College Affiliated Hospital Human Research Ethics Committee.

\section{Patient selection}

During the period January 2010 to August 2010, patients meeting the following criteria were recruited from the
Department of Radiation Oncology at the Affiliated Hospital of Guilin Medical University, Guangxi Province, China, which is located in southern China and provides health services for about 8 million people. Eligibility criteria included: patients who 1 ) were over 18 years, 2) were diagnosed with malignant tumor proven by biopsy, 3) would undergo RT with curative intent. Excluded from the study were patients who 1) had difficulty in understanding the questionnaire or communicating, 2) had a history of psychiatric disorder, 3) had distant metastasis. None of the patients died of RT-induced complications. Cancers were classified according to the 7 th edition of the tumor-node-metastasis (TNM) staging system by the American Joint Committee on Cancer (AJCC)/ Union for International Cancer Control (UICC) [21]. Informed written consent from patients was obtained for the study.

\section{Randomization}

All patients were randomly assigned to an intervention or control group. The computer-generated random allocation sequence was obtained independently by the investigators. Research nurses randomized participants 1:1 to either the control or intervention groups based on allocations before the RT.

\section{Psychosocial intervention}

The psychosocial intervention was delivered by three conductors (a clinician, a nurse and a radiation therapist) who were trained in psychotherapy techniques. Each of these people had clinical experience in RT. All participants were given a series of questionnaires to complete.

The patients randomized to the intervention group of the study received psychosocial interventions according to their meeting problems included symptoms and side effects of treatment (i.e. fatigue, nausea, vomiting and pain), and psychological issues (i.e. depression, assessed by the Zung Self-rating Depression Scale [SDS]; and anxiety, assessed by the Self-rating Anxiety Scale [SAS]).

\section{(1) Psychoeducation}

Conductors should (a) show a good medical ethic and kindly attitude to patients, which could increase safe and confident feeling, and relieve their nervous tension to make them cooperate with treatment; (b) explain the necessity of tumor radiotherapy and introduce its principles, methods, adverse reactions during radiotherapy, prevention methods and treatment for side effects, so that patients may have some knowledge of radiotherapy; (c) analyze a variety of favorable factors with patients and their relatives together, encourage patients to maintain an optimistic mood, establish the confidence to conquer disease, and cooperate with the treatment; (d) enable patients to be familiar with therapeutic environment and equipment through visiting the radiotherapy 


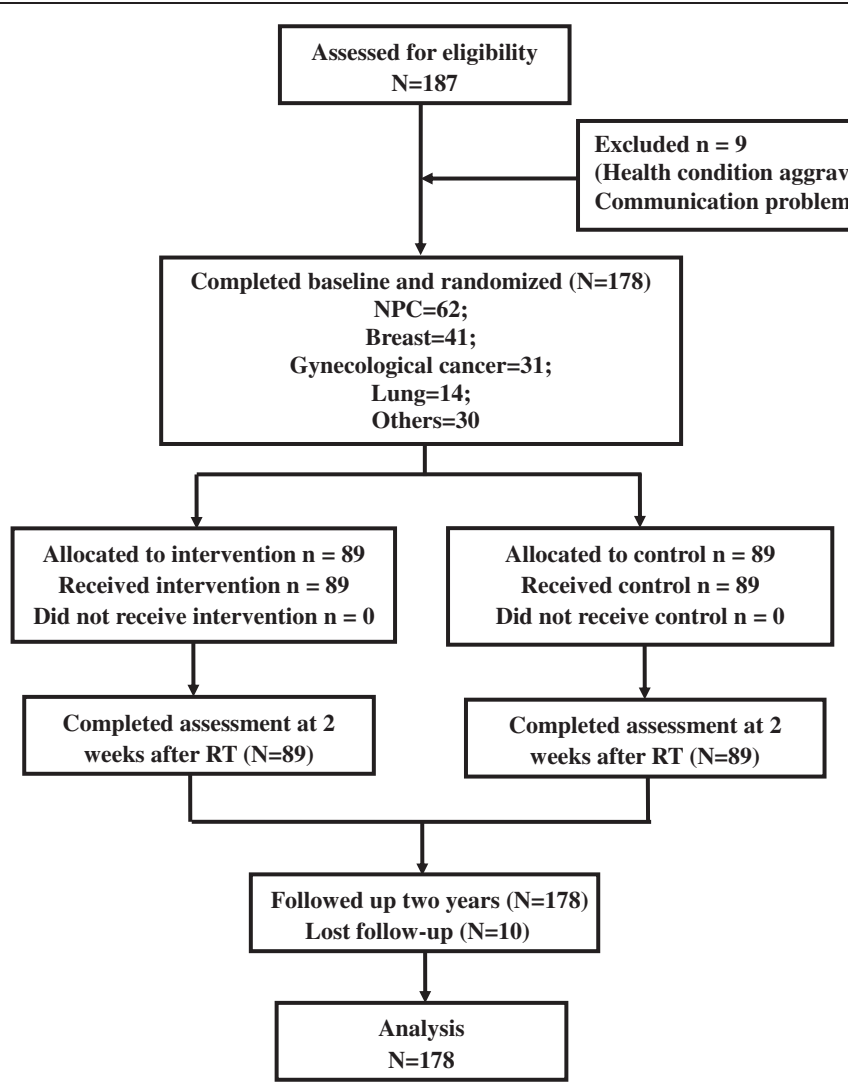

Figure 1 Patient flow and follow-up in the study.

room, and understanding the radiation process before the treatment, with the aim of reducing their fear and stress; (d) ask patients to carry out some wholesome activities during treatment, such as walking, listening to music, taking morning exercise, and so on, according to the patient's psychological characteristics, education level and hobbies, to relieve panic and nervous tension; (e) illustrate the importance of companionship and comfort to the patient's family, and strive for their cooperation, to enhance patients' confidence in the treatment and returning to society. The session was carried out by a clinician, a nurse and a radiation therapist together.

\section{(2) Cognitive-behavioral therapy (CBT)}

A CBT protocol was offered based on recommendations in the literature [22-24]. In the first stage (1-2 weeks), through the above psychological education, conductors can build a good relationship with patients, have an insight into patients' living environment, coping ability, coping styles, expectations, goals and so on, and discover any unreasonable cognitive concepts and attribution styles. In the second stage (2-3 weeks), conductors should look for facts and examples to correct patients' unreasonable cognitive concepts and attribution styles for events, and reconstruct reasonable thoughts and a positive attribution style. In the third stage (3-4 weeks), conductors can use encouragement and a behaviorstrengthening approach to consolidate the treatment effect, enable patients to have a better understanding of their attribution style through role playing, selfdirection and so on, and strengthen the positive attribution style. The session was carried out by a clinician and a nurse.

\section{(3) Supportive-expressive therapy}

The goals of the supportive-expressive therapy was to create a supportive environment in which patients were helped to face their problems, fortify their relationships and explore positive meaning in their lives [25]. The principles of supportive-expressive therapy followed a treatment manual that had been well-established in cancer populations in previous reports [26]. Therapists provided a comfortable and safe environment to encourage participants to communicate their thoughts and feelings directly and openly with others, and promoted family and social contacts to further understand specific feelings (e.g. fear and grief) from patients with cancer, which could aid in obtaining more support, and cope with the threat of malignant tumor. The session was carried out by a clinician and a nurse. 
The psychological intervention was provided in small cohorts ranging from 5 to 8 patients, and comprised two 60-min face-to-face interviews each week. In total, 8-12 therapy hours were delivered during RT.

Patients in the control group of the study received the usual education and medication from their therapist coordinator, in addition to RT.

\section{Outcome measures}

\section{Anxiety and depression}

The primary endpoint will be the level of anxiety and depression as assessed by the SAS [27] and SDS [28], respectively. Each of the two scales is a self-completion questionnaire that has 20 items rating the four common characteristics of depression and anxiety. Scores for each question range from 1 to 4 . The scores were counted up and multiplied by 1.25 to reach a standardized score, according to the instructions that accompany the scales. These questionnaires have previously been used in the Chinese population [29,30]. In accordance with the Chinese norm, a score of 50 or more on the SAS categorized individuals as having anxiety, and a score of more than 53 on the SDS categorized individuals as having depression. Higher scores indicate a greater psychological morbidity.

\section{Quality of life}

Secondary endpoints will include QOL and overall survival.

QOL was assessed with the European Organization for Research and Treatment of Cancer Quality of Life Questionnaire-Core 30 (EORTC QLQ-C30), which is a reliable and valid instrument that is widely used for measuring QOL in cancer patients. EORTC QLQ-C30 comprises 30 questions related to the functioning and symptoms of cancer patients [31,32], including five functional scales (physical, role, cognitive, emotional and social), three symptom scales (fatigue, pain, and nausea and vomiting), one global health and QOL scale, and six symptom items (dyspnea,insomnia, appetite loss, constipation, diarrhea and financial difficulties). The scores of the items range from 0 to 100. The Chinese version of the EORTC QLQ-C30 has been validated [33-35]. Higher scores indicate better QOL for the functional scales and the global health scale, whereas higher scores indicate worse health for the symptom scales and items.

\section{Overall survival and follow-up}

Overall survival (OS) was measured as the time from the date of randomization to death or the date of the last observed follow-up. Disease-free survival (DFS) was measured as the time from the date of randomization to the date of an event or the last follow-up date, where an 'event' is defined as recurrence, metastasis and death due to any cause. The follow-up information was recorded by the clinicians. Follow-up visits for OS and DFS occurred every 3 months for up to 2 years; the latest follow-up data were gathered in August 2012. The median follow-up period for the entire group was 25 months (range, 5-32 months).

\section{Statistical analysis Sample size}

To establish the sample size for the study, a $10 \%$ difference in scores for the outcome measure assessed by questionnaires between the two arms of the study was considered clinically meaningful and assuming a standard deviation of 10 [36]. With 65 patients in each group based on power calculations, there would be $80 \%$ power to detect a difference in scores at a 5\% significance level. Each of the secondary outcomes is also powered at this level. Because of the poor prognosis for cancer patient we need to add our enrolment by $30 \%$ to account for attrition. Therefore, in total we will aim to recruit 170 patients.

\section{Statistical analyses}

Clinical and demographic variables were recorded in appropriate categories, and differences between the intervention group and the control group were assessed using chi-square tests or Fisher's exact test. Student's T test was used to compare sample means for study variables (anxiety, depression and QOL). OS was analyzed using Kaplan Meier survival analyses, and compared with the two-sided log-rank test; a two-sided $p$ value < 0.05 was considered statistically significant. The statistical analyses were conducted with the software packages of SPSS (version 13.0).

\section{Results}

\section{Patient characteristics}

A total of 187 patients were consecutively enrolled in the trial. Of those, 6 patients were unable to complete the questionnaires because of aggravation of their health condition, and a further 3 patients could not be scheduled because of communication problems. The remaining 178 patients completed the assessments at baseline; the average age of the patients was 47 years, and those of women were 45 years and men were 48 years, respectively. All patients were randomly assigned to an individually tailored intervention $(n=89)$ or control $(n=89)$ group. The distribution of the major patient characteristics, treatment modalities and RT parameters was comparable for two groups' participants (Table 1). None of the patient characteristics listed in Table 1 was significantly different at the 0.05 level between two groups. 
Table 1 Sociodemographic and clinical characteristics of the patients

\begin{tabular}{|c|c|c|c|c|}
\hline Characteristic & Total $n=178(\%)$ & IG $n=89(\%)$ & $\operatorname{CON} n=89(\%)$ & $p$ value \\
\hline \multicolumn{5}{|l|}{ Sex } \\
\hline Male & $75(42 \%)$ & $38(43 \%)$ & $37(42 \%)$ & 0.879 \\
\hline Female & $103(58 \%)$ & $51(57 \%)$ & $52(58 \%)$ & \\
\hline \multicolumn{5}{|l|}{ Age (years) } \\
\hline $20-49$ & $113(63 \%)$ & $56(63 \%)$ & $57(64 \%)$ & 0.585 \\
\hline $50-69$ & $64(36 \%)$ & $33(37 \%)$ & $31(35 \%)$ & \\
\hline $70-79$ & $1(1 \%)$ & 0 & $1(1 \%)$ & \\
\hline \multicolumn{5}{|l|}{ Marital status } \\
\hline Married & $172(97 \%)$ & $87(98 \%)$ & $85(96 \%)$ & 0.406 \\
\hline Single/divorced/widow & $6(3 \%)$ & $2(2 \%)$ & $4(4 \%)$ & \\
\hline \multicolumn{5}{|l|}{ Education level } \\
\hline Primary school & $5(3 \%)$ & $2(2 \%)$ & $3(3 \%)$ & 0.808 \\
\hline Junior high school & $18(10 \%)$ & $8(9 \%)$ & $10(11 \%)$ & \\
\hline Senior high school & $86(48 \%)$ & $46(52 \%)$ & $40(45 \%)$ & \\
\hline Above college & $69(39 \%)$ & $33(37 \%)$ & $36(41 \%)$ & \\
\hline \multicolumn{5}{|l|}{ Employment status } \\
\hline Employed & $131(73 \%)$ & $67(75 \%)$ & $64(72 \%)$ & 0.707 \\
\hline Resigned & $19(11 \%)$ & $10(11 \%)$ & $9(10 \%)$ & \\
\hline Unemployed & $28(16 \%)$ & $12(13 \%)$ & $16(18 \%)$ & \\
\hline \multicolumn{5}{|l|}{ Locality } \\
\hline Urban & $78(44 \%)$ & $40(45 \%)$ & $38(43 \%)$ & 0.763 \\
\hline Rural & $100(56 \%)$ & $49(55 \%)$ & $51(57 \%)$ & \\
\hline \multicolumn{5}{|l|}{ Primary cancer site } \\
\hline Nasopharyngeal & $62(35 \%)$ & $31(35 \%)$ & $31(35 \%)$ & 0.989 \\
\hline Breast & $41(23 \%)$ & $19(21 \%)$ & $22(25 \%)$ & \\
\hline Gynecological cancer (cervical and endometrial) & $31(17 \%)$ & $14(16 \%)$ & $17(19 \%)$ & \\
\hline Lung & $14(7 \%)$ & $7(8 \%)$ & $7(8 \%)$ & \\
\hline Others (rectum Vymphomalglioma etc.) & $30(17 \%)$ & $15(17 \%)$ & $15(17 \%)$ & \\
\hline \multicolumn{5}{|l|}{ ECOG performance status } \\
\hline $0 \sim 1$ & $170(96 \%)$ & $86(97 \%)$ & $84(94 \%)$ & 0.469 \\
\hline 2 & $8(4 \%)$ & $3(3 \%)$ & $5(6 \%)$ & \\
\hline \multicolumn{5}{|l|}{ Disease progression } \\
\hline Advanced & $111(62 \%)$ & $53(60 \%)$ & $58(65 \%)$ & 0.439 \\
\hline Early stage & $67(38 \%)$ & $36(40 \%)$ & $31(35 \%)$ & \\
\hline \multicolumn{5}{|l|}{ History of operation } \\
\hline Yes & $87(49 \%)$ & $44(49 \%)$ & $43(48 \%)$ & 0.881 \\
\hline No & $91(51 \%)$ & $45(51 \%)$ & $46(52 \%)$ & \\
\hline \multicolumn{5}{|l|}{ Radiotherapy dose } \\
\hline$\geq 70 G y$ & $78(44 \%)$ & $39(44 \%)$ & $39(44 \%)$ & 0.894 \\
\hline $50 \sim 70 \mathrm{~Gy}$ & $97(54 \%)$ & $49(55 \%)$ & $48(54 \%)$ & \\
\hline$<50 G y$ & $3(1 \%)$ & $1(1 \%)$ & $2(2 \%)$ & \\
\hline Brachytherapy & $14(7 \%)$ & $6(7 \%)$ & $8(9 \%)$ & \\
\hline \multicolumn{5}{|l|}{ Chemotherapy } \\
\hline None & $52(29 \%)$ & $29(33 \%)$ & $23(26 \%)$ & 0.710 \\
\hline
\end{tabular}


Table 1 Sociodemographic and clinical characteristics of the patients (Continued)

\begin{tabular}{|c|c|c|c|c|}
\hline Neoadjuvant & $13(7 \%)$ & $6(7 \%)$ & $7(8 \%)$ & \\
\hline Concomitant & $77(43 \%)$ & $35(39 \%)$ & $42(47 \%)$ & \\
\hline Adjuvant & $102(57 \%)$ & $50(56 \%)$ & $52(58 \%)$ & \\
\hline \multicolumn{5}{|l|}{ Comorbid condition } \\
\hline None & $158(89 \%)$ & $77(87 \%)$ & $81(91 \%)$ & 0.877 \\
\hline Diabetes & $7(4 \%)$ & $4(4 \%)$ & $3(3 \%)$ & \\
\hline Hypertension & $14(8 \%)$ & $8(9 \%)$ & $6(7 \%)$ & \\
\hline Coronary heart disease & $3(2 \%)$ & $1(1 \%)$ & $2(2 \%)$ & \\
\hline Hepatitis B & $3(2 \%)$ & $2(2 \%)$ & $1(1 \%)$ & \\
\hline
\end{tabular}

Abbreviations: IG intervention group, $C O N$ control group.

\section{Main outcome measure}

\section{Anxiety and depression in patients}

At baseline, a high proportion of enrolled patients were affected by anxiety (52\%) and depression (48\%), as assessed by SAS and SDS, respectively. Of these patients, women suffered from more anxiety (61\%) and depression (53\%) than men (anxiety, 39\%; depression, 38\%) (see Additional file 1). Female patients had a higher level of anxiety and depression compared with male patients, and differences were statistically significant. (see Additional file 2). Before radiation treatment, mean anxiety scores were $53.73(\mathrm{SD}=11.88)$ and mean depression scores were $55.44(\mathrm{SD}=9.18)$ in the intervention group, versus $52.63(\mathrm{SD}=9.21)$ and 54.53 $(\mathrm{SD}=8.30)$ in the control group, respectively. No significant variation in the scores of patients with anxiety $(p=0.492)$ and depression $(p=0.489)$ in the two arms was found before randomization. After RT, patients with anxiety $(55.69, \mathrm{SD}=10.01)$ and depression $(59.05, \mathrm{SD}=9.41)$ in the control group had significantly higher scores than patients with anxiety $(48.78, \mathrm{SD}=8.95)$ and depression (51.48, SD $=7.54)$ in the intervention group $(p<0.001)$. Compared to control group patients, patients who had received a psychosocial intervention showed significantly lower scores of anxiety and depression $(p<0.001)$ after RT (Table 2).

With stratified analysis, the data shown that the enhancement of psychological wellbeing of female patients were more significant than male patients $(p<0.001)$ (see Additional file 3 ). In addition, psychological symptoms with patients underwent adjuvant chemotherapy were improved obviously compared with the other chemotherapeutic groups $(p<0.001)$. (see Additional file 4).

\section{Secondary outcomes}

\section{Quality of life in patients}

The mean QOL scores of the EORTC QLQ-C30 subscale before and after RT are shown in Table 3; higher scores reflect better QOL. Most of the subscale measures tended to become worse in the survey period. The decrease in QOL from baseline to post-RT was greater in the control arm, although the difference was not significant.

Before the start of RT, there were few significant differences between the scores of the intervention and control groups. However, compared the QOL between women and men, we found that women always had lower QOL scores than men in our sample. Such as the scores of physical functioning (PF), emotional functioning (EF), cognitive functioning (CF), global health status (QL), etc. in females, were worse than those of males $(p<0.05)$ (see Additional file 5). After completion of RT, the two groups showed statistically significant differences in terms of global health status $(p<0.001)$. In relation to functional scales, physical functioning and emotional functioning scores were higher in the intervention group than in the control group $(p<0.01)$. In relation to symptom scales/items, significant improvement was seen in insomnia $(p=0.04)$ in the intervention group. Other items scores on the EORTC QOL instrument in the

Table 2 Comparisons of SAS, SDS at the baseline and 2 weeks post-RT between two groups $(n=178)$

\begin{tabular}{|c|c|c|c|c|c|c|c|c|}
\hline & \multicolumn{4}{|c|}{ Baseline } & \multicolumn{4}{|c|}{2 weeks post-RT } \\
\hline & IG $(n=89)$ & $\operatorname{CON}(n=89)$ & & & IG $(n=89)$ & $\operatorname{CON}(n=89)$ & & \\
\hline & Mean(SD) & Mean(SD) & $t$ value & $p$ value & Mean(SD) & Mean(SD) & $t$ value & $p$ value \\
\hline SAS scores & $53.73(11.88)$ & $52.63(9.21)$ & 0.689 & 0.492 & 48.78(8.95) & $55.69(10.01)$ & -4.85 & $<0.001$ \\
\hline SDS scores & $55.44(9.18)$ & $54.53(8.30)$ & 0.693 & 0.489 & $51.48(7.54)$ & $59.05(9.41)$ & -5.92 & $<0.001$ \\
\hline
\end{tabular}

Abbreviations: IG intervention group, CON control group, SD standard deviation; RT, radiation treatment, SAS Self-rating Anxiety Scale, SDS Self-rating Depression Scale. 
Table 3 Comparisons of QOL at the baseline and 2 weeks post-RT between the two groups $(n=178)$

\begin{tabular}{|c|c|c|c|c|c|c|c|c|c|}
\hline \multirow[t]{3}{*}{ EORTC QLQ-C30 subscales } & \multirow[t]{3}{*}{ Item } & \multicolumn{4}{|c|}{ Baseline } & \multicolumn{4}{|c|}{2 weeks post-RT } \\
\hline & & \multirow{2}{*}{$\begin{array}{l}\text { IG }(\mathrm{n}=89) \\
\text { Mean(SD) }\end{array}$} & \multicolumn{3}{|l|}{$\operatorname{CON}(n=89)$} & \multirow{2}{*}{$\begin{array}{l}\text { IG }(n=89) \\
\text { Mean(SD) }\end{array}$} & \multicolumn{2}{|l|}{$\operatorname{CON}(n=89)$} & \multirow[b]{2}{*}{$p$ value* } \\
\hline & & & Mean(SD) & $t$ value & $p$ value & & Mean(SD) & $t$ value & \\
\hline \multicolumn{10}{|l|}{ Functioning scales } \\
\hline Physical functioning PF & $1-5$ & $77.23(10.19)$ & $78.91(10.81)$ & -1.067 & 0.288 & $79.70(9.80)$ & $75.36(9.71)$ & 2.970 & 0.003 \\
\hline Role functioning RF & 6,7 & $59.55(24.48)$ & $58.28(24.49)$ & 0.347 & 0.729 & $59.18(24.23)$ & $57.45(21.01)$ & 0.507 & 0.613 \\
\hline Emotional functioning $\mathbf{E F}$ & $21-24$ & $72.57(12.51)$ & $70.77(14.71)$ & 0.874 & 0.383 & $74.18(11.43)$ & $66.03(14.00)$ & 4.251 & $<0.001$ \\
\hline Cognitive functioning CF & 20,25 & $80.71(11.48)$ & $81.27(11.46)$ & -0.327 & 0.744 & $81.09(12.36)$ & $78.58(10.01)$ & 1.489 & 0.138 \\
\hline Social functioning SF & 26,27 & $75.00(13.82)$ & $73.43(12.31)$ & 0.802 & 0.424 & $74.35(10.67)$ & $71.33(11.88)$ & 1.782 & 0.076 \\
\hline Global health status QL & 29,30 & $61.31(13.23)$ & $58.43(12.78)$ & 1.48 & 0.141 & $58.80(12.20)$ & $51.12(12.82)$ & 4.092 & $<0.001$ \\
\hline \multicolumn{10}{|l|}{ Symptom scales and/or items } \\
\hline Fatigue $\mathbf{F A}$ & $10,12,18$ & $28.05(14.75)$ & $28.63(13.38)$ & -0.276 & 0.783 & $29.20(11.86)$ & $32.93(13.78)$ & -1.938 & 0.054 \\
\hline Nausea/vomiting NV & 14,15 & $11.05(11.50)$ & $9.74(9.99)$ & 0.812 & 0.418 & $14.61(12.26)$ & 15.92(10.93) & -0.753 & 0.452 \\
\hline Pain PA & 9,19 & $31.46(13.86)$ & $30.52(14.70)$ & 0.437 & 0.662 & $28.84(15.64)$ & $30.90(16.77)$ & -0.847 & 0.398 \\
\hline Dyspnea DY & 8 & $12.36(16.19)$ & $14.79(16.56)$ & -0.992 & 0.323 & $11.98(16.08)$ & $12.36(16.95)$ & -0.151 & 0.880 \\
\hline Insomnia SL & 11 & $29.21(22.37)$ & $30.71(21.45)$ & -0.456 & 0.649 & $27.71(20.25)$ & $34.08(20.71)$ & -2.074 & 0.040 \\
\hline Appetite loss AP & 13 & $23.03(22.96)$ & $25.09(22.63)$ & -0.603 & 0.547 & $26.78(21.26)$ & $28.84(23.13)$ & -0.618 & 0.537 \\
\hline Constipation CO & 16 & $16.85(20.18)$ & $16.10(20.17)$ & 0.248 & 0.805 & $18.73(19.43)$ & 20.97(19.71) & -0.766 & 0.445 \\
\hline Diarrhea DI & 17 & 10.86(19.32) & $9.36(15.88)$ & 0.565 & 0.573 & $13.11(17.13)$ & $12.36(18.38)$ & 0.281 & 0.779 \\
\hline Financial difficulties Fl & 28 & $62.17(29.38)$ & $61.42(29.26)$ & 0.170 & 0.865 & $68.17(30.11)$ & $67.04(28.65)$ & 0.255 & 0.799 \\
\hline
\end{tabular}

Abbreviations: IG intervention group, CON control group, SD standard deviation, RT radiation treatment; EORTC QLQ-C30 European Organization for Research and Treatment of Cancer Quality of Life Questionnaire Core 30 items.

* $p$ values in boldface indicate statistically significant difference.

intervention arm indicated a trend towards improvement in comparison with the control arm. However, none of these changes attained statistical significance. Financial difficulties scores changed (but not significantly) in patients before and after RT (Table 3).

According to subgroup analysis, the result indicated that the improvement of $\mathrm{QOL}$ (i.e. $\mathrm{PE}, \mathrm{EF}, \mathrm{QL}$, etc.) in female patients were more remarkable than male patients (see Additional file 6). Also, QOL of patients who received high dose radiotherapy (see Additional file 7) or underwent adjuvant chemotherapy (see Additional file 8) was significantly superior to that in the low dose radiotherapy group or other chemotherapeutic groups.

\section{Psychosocial intervention and survival}

The main OS and DFS analysis included the 178 patients. A total of 29 patients (16\%) died - $15(17 \%)$ in the intervention arm and $16(18 \%)$ in the control arm; 10 patients $(6 \%)$ were lost to follow-up - 4 (4\%) in the intervention arm and $6(7 \%)$ in the control arm. Diseasefree survival rates at 2 years were $79.8 \%$ for the intervention arm and $76.4 \%$ for the control arm (two-sided $\log$-rank, $p=0.527$; Figure 2A). The 2-year overall survival rates were $83.1 \%$ for the intervention arm and $84.3 \%$ for the control arm (two-sided log-rank, $p=0.925$;
Figure 2B). At 2 years follow-up, there was no improvement in DFS and OS rate in the intervention arm compared with the control arm.

In the subgroup analysis, life table estimates of 2-year DFS for intervention group and control group were $90.3 \%$ and $87.1 \%$ (two-sided log-rank, $p=0.703$ ) in nasopharyngeal carcinoma patients, $85.9 \%$ and $77.4 \%$ (two-sided logrank, $p=0.470$ ) in breast cancer patients, $70.1 \%$ and $87.8 \%$ (two-sided log-rank, $p=0.207$ ) in gynecological cancer patients, respectively. 2-year OS were $90.3 \%$ and $90.3 \%$ (twosided log-rank, $p=1.000$ ) in nasopharyngeal carcinoma patients, $90.7 \%$ and $88.5 \%$ (two-sided log-rank, $p=0.851$ ) in breast cancer patients, $85.1 \%$ and $94.1 \%$ (two-sided logrank, $p=0.436)$ in gynecological cancer patients, respectively (Figure $2 \mathrm{C}-\mathrm{H})$.

\section{Discussion}

The results of this randomized trial demonstrate that a psychosocial intervention significantly reduced levels of depression and anxiety compared to a control group. Further, the intervention was effective for improving elements of QOL, such as global health status and physical functioning; it also increased emotional functioning, significantly decreased insomnia, and was similar in costeffectiveness in comparison with usual care. The subgroup 

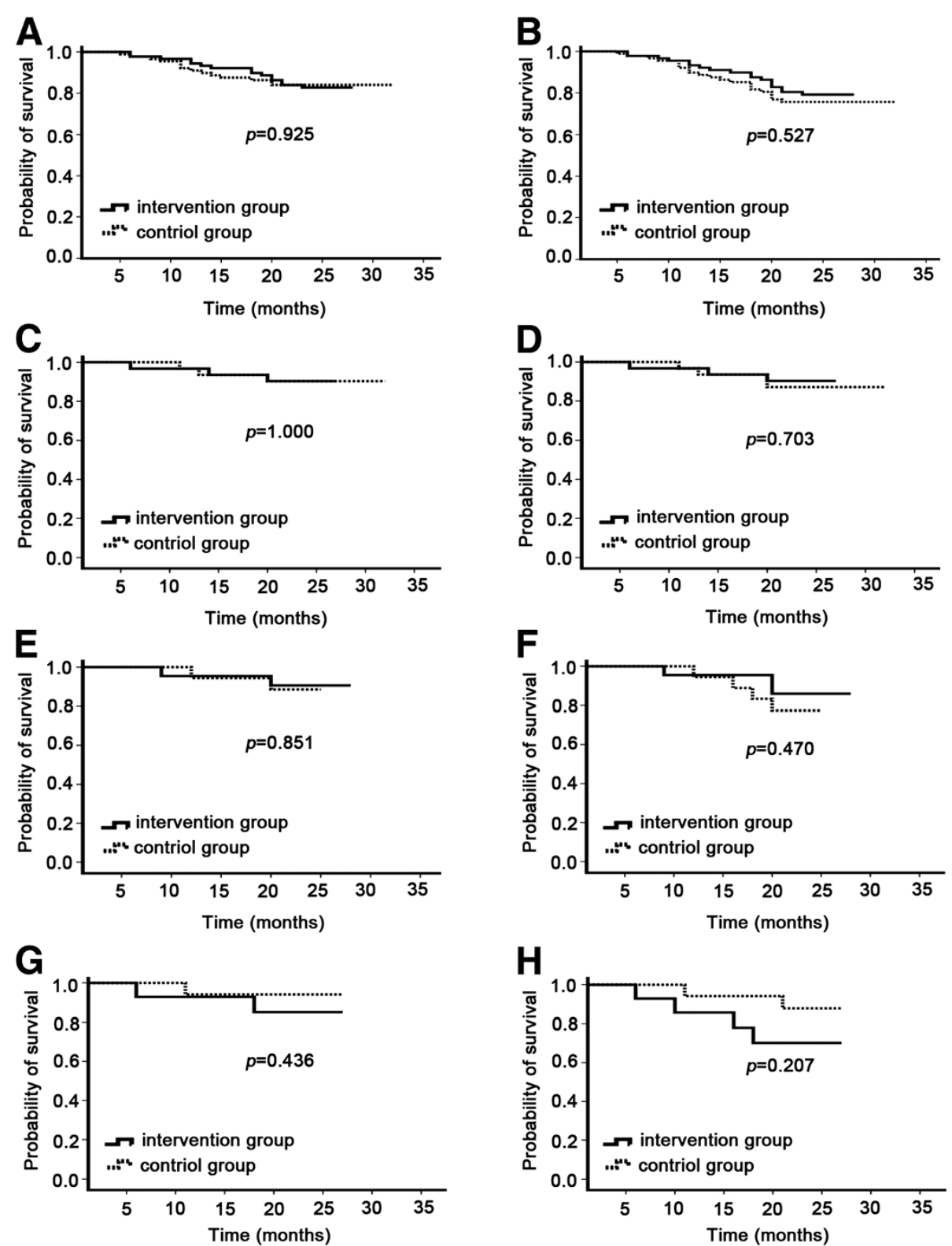

Figure 2 Survival analysis in patients randomly assigned to intervention group or control group. Kaplan-Meier survival curve for the overall survival in all patients (A), nasopharyngeal carcinoma (C), breast cancer (E) and gynecological cancer (G). Kaplan-Meier survival curve for the time to progression in all patients (B), nasopharyngeal carcinoma (D), breast cancer $(\mathbf{F})$ and gynecological cancer $(\mathbf{H})$.

analysis suggested that female patients and patients received high dose radiotherapy or underwent adjuvant chemotherapy would benefit more from the intervention. However, the intervention was not effective in prolonging survival.

More than half of the patients in our study undergoing RT for the various types of cancer expressed symptoms of depression or anxiety. This prevalence is consistent with previous reports of symptoms of psychosocial problems that ranged between $30 \%$ and $70 \%$ [37,38]. There were significant gender differences between anxiety and depression in our sample, and the female patients were generally more anxious and depressed compared to male patients. This result complied with Massie's findings [39]. The reason for the appearance of negative moods in participants could be due to trepidation about a poor prognosis of cancer, misunderstanding of RT and worry about adverse effects of RT. In particular, for women with cancers, more misgivings were here compared to men, including fear of the diseases would impact their attractiveness, sexual relationships, fertility and even family happiness $[40,41]$. Despite a high prevalence of 
mental ill-health following the diagnosis of cancer, little effort has been applied to meeting such needs [42,43]. Therefore, it is imperative to assess the mental health of patients and take some measures to alleviate anxiety and depression throughout the process of RT.

The results of a psychosocial intervention in this study were encouraging. Following the psychosocial intervention, significant differences in depression and anxiety between two groups were observed after the completion of RT. A marked decrease in levels of depression and anxiety occurred within the intervention group. However, in the control group the results showed a trend for a deterioration. Thus, we could speculate that daily anticancer treatment will aggravate psychiatric distress if the patient does not also receive psychological care and provision of support from medical personnel during RT. The findings were in line with those reported in other studies. For example, Goerling $\mathrm{U}$ et al., using random sample analyses, reported an increase in the psychological condition of patients with cancer on a surgical ward after patients underwent psycho-oncological support [44]. Faul LA et al. designed a randomised study to evaluate the effectiveness of skill in stress management for cancer patients receiving chemotherapy. The authors demonstrated that psychosocial care was an efficient approach that clearly reduced anxiety and depression in patients with cancer [45]. Arguably, it is acceptable to define tailored psychological support plans whenever needed, with the aim of preventing or managing emotional problems appearing in RT.

This study also evaluated differences in QOL in a large sample of cancer patients, with and without intervention during RT. The results showed a trend for a deterioration in the control group compared to a stabilization in the intervention group. After RT, patients in the intervention group achieved significantly higher scores for global health status, physical and emotional functioning, and improvement in insomnia than patients in the control group. Therefore interventions are more necessary for these patients. Our findings agree with those from a randomized controlled trial conducted by Breitbart W, who found that participants who received psychotherapy demonstrated significantly greater improvement than the control group in terms of spiritual well-being and QOL [46]. Similarly, Eom CS et al. investigated the association between mental health, QOL and perceived social support in 1930 patients with cancer recruited from multiple centers and found that interventions improved mental health and QOL in cancer patients through a direct effect [47]. Moreover, our data showed that there was no significant difference in the financial difficulties subscale between the intervention and control groups at assessment after RT. Mean costs in the intervention group were CNY45,000, and were not higher than for the control group receiving usual care. The findings were similar to previous studies [48], and suggested that a psychological support during RT could be a cost-effective tool for improving QOL in patients with cancer.

Unplanned subgroup analysis demonstrated that obvious associations between psychological distress or QOL and clinical characteristics (including gender, radiation dose and chemotherapy modes) among oncology patients. A possible reason may be that women possessed more misgivings and trepidation, and high dose radiotherapy or adjuvant chemotherapy always resulted in severely toxic side effect, which significantly impacts mental status and QOL of patients. Thus, when screening of mental health and QOL in cancer patients who undergoing radiotherapy, female patients, those that received high dose radiotherapy, and those that underwent adjuvant chemotherapy should be routinely emphasized, who would benefit more from the psychosocial intervention.

Survival analyses from this trial indicated that patients with cancer randomized to receive a psychosocial intervention had no reduction in their risk for cancer recurrence and death compared to those who did not receive the intervention. An earlier finding that intervention was correlated with longer survival was not replicated [20]. Our results are in accordance with the literature showing that interventions for patients with cancer did not extended survival times $[26,49,50]$. Our results demonstrated that beneficial effects on survival is the major reason for patients receiving more anti-cancer treatment, and the key benefit of psychotherapeutic interventions is improved psychological well-being. However, the survival debate continues.

There is no consensus on how to define the psychological symptoms and problems of QOL in patients with cancer, and therapists always find it difficult to know what tests to order and which patients to treat, when and how long to treat, and what the available treatment options are. During the therapeutic process in our study, we observed that many factors could affect the moods and QOL, and that different patients varied in their receptiveness to psychosocial interventions. Although some of these factors cannot be avoided, psychosocial intervention effectively achieved benefits for patients. Therefore, as Simon Wein has stated, it is essential that clinical staff in oncology departments gain some knowledge of psycho-oncology, including communication skills, psychotropic medications and psychological therapies in routine clinical practice, so that they can clinically identify distress and provide initial psychosocial support if necessary [51].

Our trail has some potential limitations. First, duration of time of the survey for anxiety, depression and QOL was short, which meant that we were unable to determine 
what changes of moods and QOL in cancer patients would take place in the time after the end of RT. Second, survival following RT is often short, so the results may not exactly reflect the relationship between intervention therapy and survival. Finally, this study was performed using a single-center design and the sample size was relatively small, which may cause potential sampling errors. To investigate the usefulness and feasibility of intervention, further work, including a prospective longitudinal multicenter study, is recommended.

\section{Conclusion}

With the use of new RT techniques (such as intensitymodulated radiation therapy [IMRT]) and multimodal treatment regimes in oncology practice, an increase in survival rates over the last few decades [52] had led to a greater proportion of patients calling for higher QOL after cancer treatment. The effect of individuals' psychological well-being have become relevant parameters in oncology research and practice. The results of this randomized trial demonstrated that a psychosocial intervention during RT for patients with cancer is a practical, cost-effective tool for helping most patients receiving RT to reduce anxiety, depression and improve their QOL. The intervention was easy to implement during $\mathrm{RT}$ in hospital using health professionals (a clinician, a nurse, a radiation therapist ) who had undergone simple training in psychotherapy techniques, and that the treatment input required was minimal, which facilitated uptake by patients with cancer. Overall, psychosocial care during RT, as an important cure strategy, should be carried out in routine clinical practice.

\section{Additional files}

\section{Additional file 1: Table S1. The prevalence (\%) of women and men} suffering from anxiety and depression.

Additional file 2: Table S2. Comparisons of SAS and SDS between women and men at the baseline $(n=178)$.

Additional file 3: Table S3. Comparisons of SAS and SDS at the baseline and 2 weeks post-RT in male and female patients for subanalysis $(n=178)$.

Additional file 4: Table S4. Comparisons of SAS and SDS at the baseline and 2 weeks post-RT in in different chemotherapy for subanalysis ( $n=178)$.

Additional file 5: Table S5. Comparisons of QOL between women and men at the baseline $(n=178)$.

Additional file 6: Table S6. Comparisons of QOL at the baseline and 2 weeks post-RT in male and female patients for subanalysis $(n=178)$.

Additional file 7: Table S7. Comparisons of QOL at the baseline and 2 weeks post-RT in different dose of radiotherapy for subanalysis $(n=178)$.

Additional file 8: Table S8. Comparisons of $\mathrm{QOL}$ at the baseline and 2 weeks post-RT in different chemotherapy for subanalysis $(n=178)$.

\section{Abbreviations}

QOL: Quality of life; RT: Radiation therapy; SDS: The zung self-rating depression scale; SAS: The self-rating anxiety scale; EORTC QLQ-C30) (QLQC30[3.0]: The European Organization for research and treatment of cancer quality of life questionnare-core 30; TNM: Tumor-node-metastasis; AJCC: The American joint committee on cancer; UICC: Union for international cancer control; CBT: Cognitive-behavioral therapy; OS: Overall survival; DFS: Diseasefree survival; IG: Intervention group; CON: Control group; SD: Standard deviation; ECOG: The Eastern Cooperative oncology group.

\section{Competing interests}

The authors have no actual or potential conflicts of interest to declare.

\section{Authors' contributions}

ZG and HYT conceived the project, participated in its design and performed psychosocial intervention and led manuscript development; HL provided methodological guidance and intellectual comment for psychosocial intervention, and helped to draft the manuscript; SKT performed the statistical analysis and guided interpretation of results; KHF and YCH participated in its design and coordination; QB provided expertise on chemotherapy and helped to draft the manuscript; WJ directed the study, its design, performed psychosocial intervention, and drafted the manuscript. All authors read and approved the final manuscript.

\section{Authors' information}

First author: share first authorship Zhen Guo and Hua-ying Tang.

\section{Acknowledgment}

We thank the patients who participated in this trial and we are also grateful to clinical staff who helped with patient recruitment, and research associates who facilitated submission of patient-completed questionnaires. This study was funded by grants from the Health Bureau of Guangxi Province of China (No.Z2010279) and Guangxi Medical Scientific Experiment Center Open Fund (grant KFJJ2010-50).

\section{Author details}

'Department of Radiation Oncology, Guilin Medical College Affiliated Hospital, 15 Lequn Road, Guilin, People's Republic of China. ${ }^{2}$ School of Nursing, Guilin Medical College, 109 Huanchen Road North, Guilin, People's Republic of China. ${ }^{3}$ Department of Psychiatry, Guilin Medical College Affiliated Hospital, 15 Lequn Road, Guilin, People's Republic of China. ${ }^{4}$ School of Public Health, Guilin Medical College, 109 Huanchen Road North, Guilin, People's Republic of China. ${ }^{5}$ Department of Medical Oncology, Guilin Medical College Affiliated Hospital, 15 Lequn Road, Guilin, People's Republic of China.

Received: 24 February 2013 Accepted: 8 July 2013

Published: 17 July 2013

\section{References}

1. Berkey FJ: Managing the adverse effects of radiation therapy. Am Fam Physician 2010, 82(381):388-394.

2. Brix C, Schleussner C, Fuller J, Roehrig B, Wendt TG, Strauss B: The need for psychosocial support and its determinants in a sample of patients undergoing radiooncological treatment of cancer. J Psychosom Res 2008, 65:541-548.

3. Derogatis $L R$, Morrow GR, Fetting J, Penman D, Piasetsky S, Schmale AM, Henrichs M, Carnicke CL Jr: The prevalence of psychiatric disorders among cancer patients. JAMA 1983, 249:751-757.

4. Fallowfield L, Ratcliffe D, Jenkins V, Saul J: Psychiatric morbidity and its recognition by doctors in patients with cancer. $\mathrm{Br} J$ Cancer 2001, 84:1011-1015.

5. Brintzenhofe-Szoc KM, Levin TT, Li Y, Kissane DW, Zabora JR: Mixed anxiety/ depression symptoms in a large cancer cohort: prevalence by cancer type. Psychosomatics 2009, 50:383-391.

6. Hinz A, Krauss O, Hauss JP, Hockel M, Kortmann RD, Stolzenburg JU, Schwarz R: Anxiety and depression in cancer patients compared with the general population. Eur J Cancer Care (Engl) 2010, 19:522-529.

7. Linden W, Vodermaier A, Mackenzie R, Greig D: Anxiety and depression after cancer diagnosis: Prevalence rates by cancer type, gender, and age. J Affect Disord 2012, 141:343-351.

8. Pugliese P, Perrone M, Nisi E, Garufi C, Giannarelli D, Bottomley A, Terzoli E: An integrated psychological strategy for advanced colorectal cancer patients. Health Qual Life Outcomes 2006, 4:9. 
9. Breitbart W: Identifying patients at risk for, and treatment of major psychiatric complications of cancer. Support Care Cancer 1995, 3:45-60.

10. Pelletier G, Verhoef MJ, Khatri N, Hagen N: Quality of life in brain tumor patients: the relative contributions of depression, fatigue, emotional distress, and existential issues. J Neurooncol 2002, 57:41-49.

11. Lloyd-Williams M, Shiels C, Taylor F, Dennis M: Depression-an independent predictor of early death in patients with advanced cancer. J Affect Disord 2009, 113:127-132.

12. Mainio A, Hakko H, Timonen M, Niemela A, Koivukangas J, Rasanen P: Depression in relation to survival among neurosurgical patients with a primary brain tumor: a 5-year follow-up study. Neurosurgery 2005, 56:1234-1241. discussion 1241-1232.

13. Goerling U, Odebrecht S, Schiller G, Schlag PM: Need for psychosocial care in in-patients with tumour disease. Investigations conducted in a clinic specializing in tumour surgery. Chirurg 2006, 77:41-46. discussion 46.

14. Carlson LE, Angen M, Cullum J, Goodey E, Koopmans J, Lamont L, MacRae $\mathrm{JH}$, Martin M, Pelletier G, Robinson J, et al: High levels of untreated distress and fatigue in cancer patients. Br J Cancer 2004, 90:2297-2304.

15. Osborn RL, Demoncada AC, Feuerstein M: Psychosocial interventions for depression, anxiety, and quality of life in cancer survivors: metaanalyses. Int J Psychiatry Med 2006, 36:13-34.

16. Sheinfeld Gorin S, Krebs P, Badr H, Janke EA, Jim HS, Spring B, Mohr DC, Berendsen MA, Jacobsen PB: Meta-analysis of psychosocial interventions to reduce pain in patients with cancer. $J$ Clin Oncol 2012, 30:539-547.

17. Gordon LG, Beesley VL, Scuffham PA: Evidence on the economic value of psychosocial interventions to alleviate anxiety and depression among cancer survivors: a systematic review. Asia Pac J Clin Oncol 2011, 7:96-105

18. Fang CY, Reibel DK, Longacre ML, Rosenzweig S, Campbell DE, Douglas SD: Enhanced psychosocial well-being following participation in a mindfulness-based stress reduction program is associated with increased natural killer cell activity. J Altern Complement Med 2010, 16:531-538.

19. Steel $\mathrm{L}$, Nadeau K, Olek M, Carr Bl: Preliminary results of an individually tailored psychosocial intervention for patients with advanced hepatobiliary carcinoma. J Psychosoc Oncol 2007, 25:19-42.

20. Andersen BL, Yang HC, Farrar WB, Golden-Kreutz DM, Emery CF, Thornton LM, Young DC, Carson WE 3rd: Psychologic intervention improves survival for breast cancer patients: a randomized clinical trial. Cancer 2008 113:3450-3458.

21. Edge SB, Byrd DR, Compton CC, Fritz AG, Greene FL, Trotti A: American Joint Committee on Cancer Staging Manual. 7th edition. New York: Springer; 2009.

22. Lee H, Lim Y, Yoo MS, Kim Y: Effects of a nurse-led cognitive-behavior therapy on fatigue and quality of life of patients with breast cancer undergoing radiotherapy: an exploratory study. Cancer Nurs 2011, 34:E22-E30.

23. Mannix KA, Blackburn IM, Garland A, Gracie J, Moorey S, Reid B, Standart S, Scott J: Effectiveness of brief training in cognitive behaviour therapy techniques for palliative care practitioners. Palliat Med 2006, 20:579-584.

24. Cohen M, Kuten A: Cognitive-behavior group intervention for relatives of cancer patients: a controlled study. J Psychosom Res 2006, 61:187-196.

25. Kissane DW, Grabsch B, Clarke DM, Christie G, Clifton D, Gold S, Hill C, Morgan A, McDermott F, Smith GC: Supportive-expressive group therapy: the transformation of existential ambivalence into creative living while enhancing adherence to anti-cancer therapies. Psychooncology 2004, 13:755-768.

26. Goodwin PJ, Leszcz M, Ennis M, Koopmans J, Vincent L, Guther H, Drysdale E, Hundleby M, Chochinov HM, Navarro M, et al: The effect of group psychosocial support on survival in metastatic breast cancer. $N$ Engl J Med 2001, 345:1719-1726.

27. Zung WW: A rating instrument for anxiety disorders. Psychosomatics 1971, 12:371-379.

28. Zung WW: A self-rating depression scale. Arch Gen Psychiatry 1965, 12:63-70.

29. Liang T, Liu EW, Zhong H, Wang B, Shen LM, Wu ZL: Reliability and validity of addiction severity index in drug users with methadone maintenance treatment in Guizhou province, China. Biomed Environ Sci 2008, 21:308-313.
30. Li A: Analyses on the rate and epidemic characteristics of anxiety and depression among cancer patients in Yangpu District in Shanghai. Asian Pac J Cancer Prev 2009, 10:895-898.

31. Aaronson NK, Ahmedzai S, Bergman B, Bullinger M, Cull A, Duez NJ, Filiberti A, Flechtner H, Fleishman SB, De Haes JC, et al: The European Organization for Research and Treatment of Cancer QLQ-C30: a quality-of-life instrument for use in international clinical trials in oncology. $J \mathrm{Natl}$ Cancer Inst 1993, 85:365-376.

32. Efficace F, Bottomley A, Vanvoorden V, Blazeby JM: Methodological issues in assessing health-related quality of life of colorectal cancer patients in randomised controlled trials. Eur J Cancer 2004, 40:187-197.

33. Zhao H, Kanda K: Testing psychometric properties of the standard Chinese version of the European Organization for Research and Treatment of Cancer Quality of Life Core Questionnaire 30 (EORTC QLQ-C30). J Epidemiol 2004, 14:193-203.

34. Chien TW, Lin SJ, Wang WC, Leung HW, Lai WP, Chan AL: Reliability of $95 \%$ confidence interval revealed by expected quality-of-life scores: an example of nasopharyngeal carcinoma patients after radiotherapy using EORTC QLQ-C 30. Health Qual Life Outcomes 2010, 8:68.

35. Huang CC, Lien HH, Sung YC, Liu HT, Chie WC: Quality of life of patients with gastric cancer in Taiwan: validation and clinical application of the Taiwan Chinese version of the EORTC QLQ-C30 and EORTC QLQ-STO22. Psychooncology 2007, 16:945-949.

36. Turner J, Kelly B, Clarke D, Yates P, Aranda S, Jolley D, Chambers S, Hargraves $M$, McFadyen $L$ : A randomised trial of a psychosocial intervention for cancer patients integrated into routine care: the PROMPT study (promoting optimal outcomes in mood through tailored psychosocial therapies). BMC Cancer 2011, 11:48.

37. Kyranou M, Paul SM, Dunn LB, Puntillo K, Aouizerat BE, Abrams G, Hamolsky D, West C, Neuhaus J, Cooper B, Miaskowski C: Differences in depression, anxiety, and quality of life between women with and without breast pain prior to breast cancer surgery. Eur J Oncol Nurs 2013, 17:190-195.

38. Delgado-Guay M, Parsons HA, Li Z, Palmer JL, Bruera E: Symptom distress in advanced cancer patients with anxiety and depression in the palliative care setting. Support Care Cancer 2009, 17:573-579.

39. Massie MJ: Prevalence of depression in patients with cancer. J Nat/ Cancer Inst Monogr 2004, 57:71.

40. Webb C, Wilson-Barnett J: Hysterectomy: a study in coping with recovery. J Adv Nurs 1983, 8:311-319.

41. Lam WW, Au AH, Wong JH, Lehmann C, Koch U, Fielding R, Mehnert A: Unmet supportive care needs: a cross-cultural comparison between Hong Kong Chinese and German Caucasian women with breast cancer. Breast Cancer Res Treat 2011, 130:531-541.

42. Keller M, Sommerfeldt S, Fischer C, Knight L, Riesbeck M, Lowe B, Herfarth C, Lehnert $T$ : Recognition of distress and psychiatric morbidity in cancer patients: a multi-method approach. Ann Oncol 2004, 15:1243-1249.

43. Pascoe S, Edelman S, Kidman A: Prevalence of psychological distress and use of support services by cancer patients at Sydney hospitals. Aust N Z J Psychiatry 2000, 34:785-791.

44. Goerling U, Foerg A, Sander S, Schramm N, Schlag PM: The impact of short-term psycho-oncological interventions on the psychological outcome of cancer patients of a surgical-oncology department - a randomised controlled study. Eur J Cancer 2011, 47:2009-2014.

45. Faul LA, Jim HS, Williams C, Loftus L, Jacobsen PB: Relationship of stress management skill to psychological distress and quality of life in adults with cancer. Psychooncology 2010, 19:102-109.

46. Breitbart W, Poppito S, Rosenfeld B, Vickers AJ, Li Y, Abbey J, Olden M, Pessin H, Lichtenthal W, Sjoberg D, Cassileth BR: Pilot randomized controlled trial of individual meaning-centered psychotherapy for patients with advanced cancer. J Clin Oncol 2012, 30:1304-1309.

47. Eom CS, Shin DW, Kim SY, Yang HK, Jo HS, Kweon SS, Kang YS, Kim JH, Cho $\mathrm{BL}$, Park JH: Impact of perceived social support on the mental health and health-related quality of life in cancer patients: results from a nationwide, multicenter survey in South Korea. Psychooncology 2013 22:1283-1290.

48. Lemieux J, Topp A, Chappell H, Ennis M, Goodwin PJ: Economic analysis of psychosocial group therapy in women with metastatic breast cancer. Breast Cancer Res Treat 2006, 100:183-190.

49. Kissane DW, Grabsch B, Clarke DM, Smith GC, Love AW, Bloch S, Snyder RD, Li Y: Supportive-expressive group therapy for women with metastatic 
breast cancer: survival and psychosocial outcome from a randomized controlled trial. Psychooncology 2007, 16:277-286.

50. Choi J, Kuo CW, Sikorskii A, You M, Ren D, Sherwood PR, Given CW, Given $B A$ : Cognitive behavioral symptom management intervention in patients with cancer: survival analysis. Support Care Cancer 2012, 20:1243-1250.

51. Wein S, Sulkes A, Stemmer S: The oncologist's role in managing depression, anxiety, and demoralization with advanced cancer. Cancer $\int$ 2010, 16:493-499.

52. Robbins ME, Brunso-Bechtold JK, Peiffer AM, Tsien Cl, Bailey JE, Marks LB: Imaging radiation-induced normal tissue injury. Radiat Res 2012, 177:449-466.

doi:10.1186/1477-7525-11-121

Cite this article as: Guo et al:: The benefits of psychosocial interventions for cancer patients undergoing radiotherapy. Health and Quality of Life Outcomes 2013 11:121.

\section{Submit your next manuscript to BioMed Central and take full advantage of:}

- Convenient online submission

- Thorough peer review

- No space constraints or color figure charges

- Immediate publication on acceptance

- Inclusion in PubMed, CAS, Scopus and Google Scholar

- Research which is freely available for redistribution 